\title{
Demo: Software-Defined Cooking (SDC) using a Microwave Oven
}

\author{
Haojian Jin, Jingxian Wang, Swarun Kumar, Jason Hong \\ Carnegie Mellon University \\ Pittsburgh, PA, USA \\ \{haojian@cs.,jingxian@,swarun@,jasonh@cs.\}cmu.edu
}

\begin{abstract}
We present a demonstration of $S D C$ [1], a low-cost closedloop microwave oven system that aims to heat the food in a software-defined thermal trajectory. SDC achieves this through a novel high-resolution heat sensing and actuation system that uses microwave-safe components to augment existing microwaves. In this demo, we demonstrate our experimental test-bed, a modified commercial off-the-shelf microwave oven, and show a SDC-enabled microwave can be enlisted to perform unexpected cooking tasks: cooking meat and fat in bacon discriminatively and heating rice uniformly.
\end{abstract}

\section{INTRODUCTION}

So much technology now is coming into the culinary world, where cooks maybe not have to be as talented or work as hard because technology is allowing them to be better cooks than they normally might be.

David Chang, The mind of a Chef [3]

In this work, we present the demonstration of SDC, a lowcost closed-loop microwave oven system that aims to heat the food in a software-defined thermal trajectory [1].

SDC is motivated by two driving forces. First, we argue that computationally actuating the heating process can improve both cooking and dining experience. SDC would be an enabling technology for molecular gastronomy, helping chefs control the physical and chemical processes that occur while cooking. Second, among all three common heating methods (convection, conduction, and radiation), radiation is the only one that can redirect energy towards desired location (we refer the reader to [1] for a detailed analysis of related work). So Microwave oven is an ideal platform to enable software defined cooking.

Permission to make digital or hard copies of part or all of this work for personal or classroom use is granted without fee provided that copies are not made or distributed for profit or commercial advantage and that copies bear this notice and the full citation on the first page. Copyrights for thirdparty components of this work must be honored. For all other uses, contact the owner/author(s).

MobiCom '19, October 21-25, 2019, Los Cabos, Mexico

() 2019 Copyright held by the owner/author(s).

ACM ISBN 978-1-4503-6169-9/19/10.

https://doi.org/10.1145/3300061.3343368

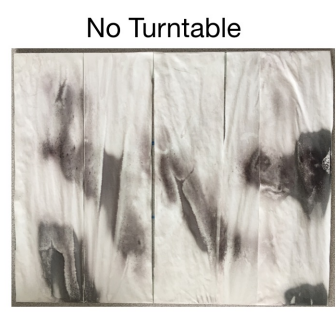

HeatFab Uniform Heating

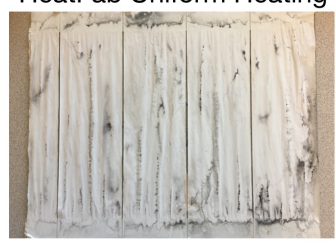

Default Turntable

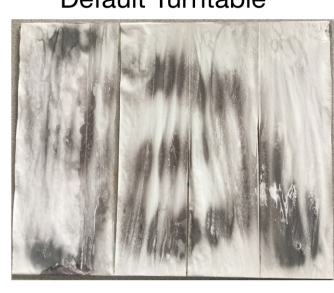

HeatFab Arbitrary Heating

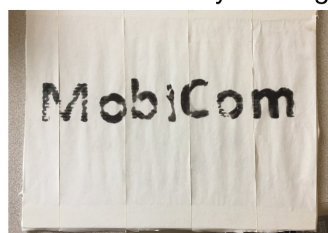

Figure 1: Results of microwaving wet thermal paper (black spots indicate high heat) in: (Top) A traditional microwave without/with turntable; (Bottom) SDC, for uniform heating (fewer black spots show heat is spread uniformly) or heating to write "MobiCom".

SDC's design can unlock numerous programmable heating opportunities (see Fig. 1). For example, when microwaving liquids (e.g. milk, baby formula), one need not worry about the uneven heating that may scald the mouth or destroy nutrients - a reason why microwaves are never advised to heat formula despite their convenience. Further, SDC can enable fine-grained forms of cooking, such as a Maillard reaction that focuses heat on the food's surface and occurs when searing meats and pan-frying vegetables.

At the heart of SDC is a novel approach both to sense and control heat at different points in space within the microwave chamber. SDC senses heat using the phenomenon that produces heat in the first place: the electro-magnetic (EM) field. SDC uses tiny RF-powered neon lights that glow in response to the EM-field within the microwave to measure the amplitude of the electromagnetic field at any given point. Upon EM field sensing, SDC then dynamically adjusts the turntable rotation and magnetron On/Off schedule to apply microwave heating hot spots to desired locations. Further, SDC uses the microwave susceptors/shields to amplify/weaken heating at a specific location to achieve more skewed peak-to-peak temperature differences. 


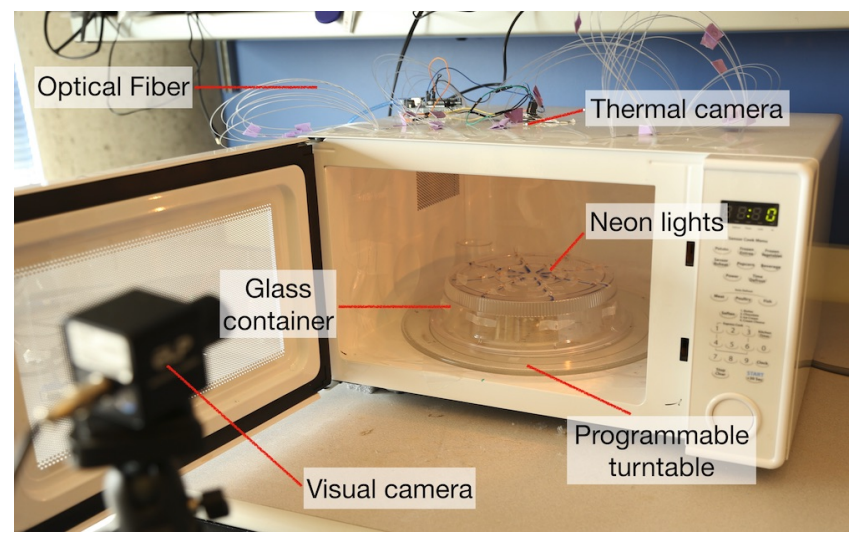

Figure 2: SDC's Hardware

In this demo, we aim to show how SDC senses the EM field in realtime and actuates the turntable accordingly to heat the food in a desired thermal trajectory. We implement a prototype of SDC by modifying a commercial Microwave oven (Sharp SMC1441CW). We place neon lamp arrays inside the microwave oven cavity and use a camera outside the cavity to monitor lamp flashes conducted via a fiber-optic cable. We replace the coarse turntable motor with a step motor controllable via an Arduino board. Given a desired heating distribution pattern, SDC recommends the initial position where the user should place the food. During the heating, SDC continuously senses the real-time EM filed strength around the food and adjusts the actuation plan. Figure 2 depicts the hardware setup of SDC.

\section{SDC'S DESIGN}

In this section, we first present the problem formulation of software-defined cooking (SDC) and then describe the design of sensing and actuation components.

Specifying SDC's Inputs, Outputs and Goal: Since microwave is essentially a heating instrument, "cooking" in a microwave is specified by three main variables:

(1) Temperature: The most important variable in cooking is the temperature of food, which will trigger different chemical reactions [2] (e.g., protein denaturation, Maillard reaction, and caramelization).

(2) Time: Time is an important factor in both cooking food accurately and killing bacteria.

(3) SpACE: Different parts of food (e.g. meat vs. fat, egg white vs. yolk) may need to be cooked with different specifications to obtain optimal tasting food [2,3]. SDC therefore aims to specify heating requirements for each spatial "pixel" of the food's surface.

In summary, we envision that the future microwave recipes specify the desired thermal trajectory, i.e. temperature vs. time, for each "pixel" of the food's surface (see Fig. 3 for an example steak recipe). This is precisely SDC's input, with its

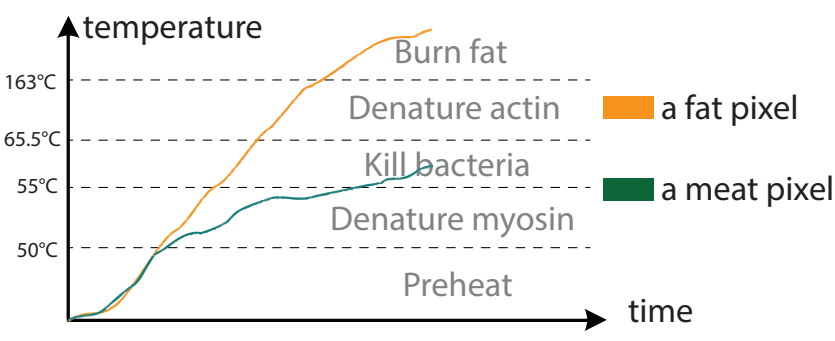

Figure 3: SDC recipe is a progression of desired temperature vs. time per-pixel of food.

performance dictated by how closely it follows this specification.

SDC's Heat Sensing: While SDC can deploy infrared cameras to sense the surface temperature of the food, doing so has two key disadvantages. First, cameras cannot sense heat in the presence of objects that blocks the food from view. Second, cameras would only measure the effect of heating after-the-fact and cannot prevent heating-damage that is already done. This motivates the need to sense "intent to heat" within the microwave rather than the current temperature of the food content.

SDC addresses this challenge by deploying microwavesafe passive electronics that senses the underlying physical phenomenon producing heat, i.e. the electromagnetic (EM) field. Specifically, we deploy neon lights that harvest EM energy in the microwave to glow in proportion to its intensity. We then measure the intensity of the light to infer field intensity and estimate the temperature distribution that would result. We do this via optical fibers that carry the visible light signal outside the chamber, allowing SDC to measure light signals from lights that are hidden from view.

SDC's Heat Actuation: Having sensed the current and estimated heat distribution over space, SDC must now actuate the control system to focus energy towards some specific areas of the food and away from others. A traditionally available instrument for heat actuation within the microwave is the turntable. Microwaves use turntables to even out heat distribution, ensuring that hotspots do not hover statically over specific areas of the food. Yet, turntables are coarse actuators given that they spread out energy blindly rather than focusing it on specific parts of the food.

In contrast, SDC engineers specific hot and cold regions of the food by building specialized microwave shields and susceptors that protect or focus specific regions of the food. We then electronically control the turntable to move food in and out of these sensed hot/cold spots as needed to cook food according to the desired heat pattern.

\section{DEMONSTRATION}

We offer two live demonstrations: uniform and arbitrary heating. 


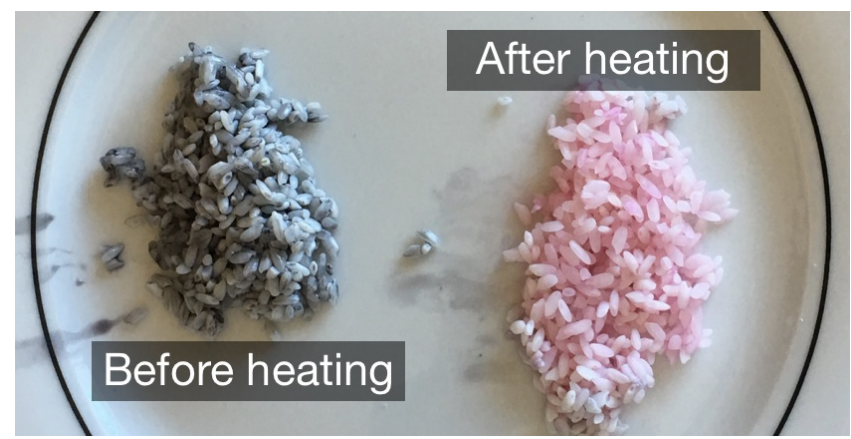

Figure 4: We color rice grains with thermal-chromatic pigment, which turn pink in a predictable manner as their temperature increases.

Demo Requirements: We need: 1) a poster board; 2) a 120-volt power outlet that supports 1000 -watt for our oven. Uniform Heating Demo Setup. This demonstration heats raw rice grains to show that SDC can heat food more uniformly than traditional microwave ovens. To visualize the heat pattern, we color the grains with thermal-chromatic pigment (Fig. 4), which will turn into pink as the temperature increases. We will heat the rice from the room temperature and set the target temperature to $60^{\circ} \mathrm{C}$ over 2-minutes of microwaving. SDC will guide the heating process using a uniform heating recipe.

To characterize the benefits of SDC, we use two blind microwave heating as the baselines: the same microwave oven (1) with and (2) without turntable rotation. Fig. 5 shows a visualization of the thermal-chromatic pigment, which changes color at $31^{\circ} \mathrm{C}$ and progresses to darker shades of pink with increased temperature. The rice colored (dull white) regions denote spots of food that remain below $31^{\circ} \mathrm{C}$. We observe that SDC achieves a uniform pink hue that darkens over time, while the baselines (no rotation or default rotation) continue to have cold spots through time.

Arbitrary Heating Demo Setup. This demonstration heats meat and fat in bacon discriminatively to show that SDC can heat food in an arbitrary thermal pattern. We use the heating recipe required per pixel for the meat vs. the fat as shown in Fig. 3. Based on the package instruction, we set the heating time to 1 minute. We place strips of bacon on the microwave plate across multiple experiments.

Fig. 6 depicts the initial and final product over the cooking process. These three slices of bacon are from the same package, so the original fat patterns are nearly identical. Heated meat and fat will shrink. SDC applies heat to meat and fat discriminatively, so the fat shrinks more than the meat. Note that while default rotation heats the bacon unevenly (resulting in the uneven shape), SDC heats the microwave more uniformly while differentiating between the heat applied to meat and fat. Indeed, we observe that the cooking process

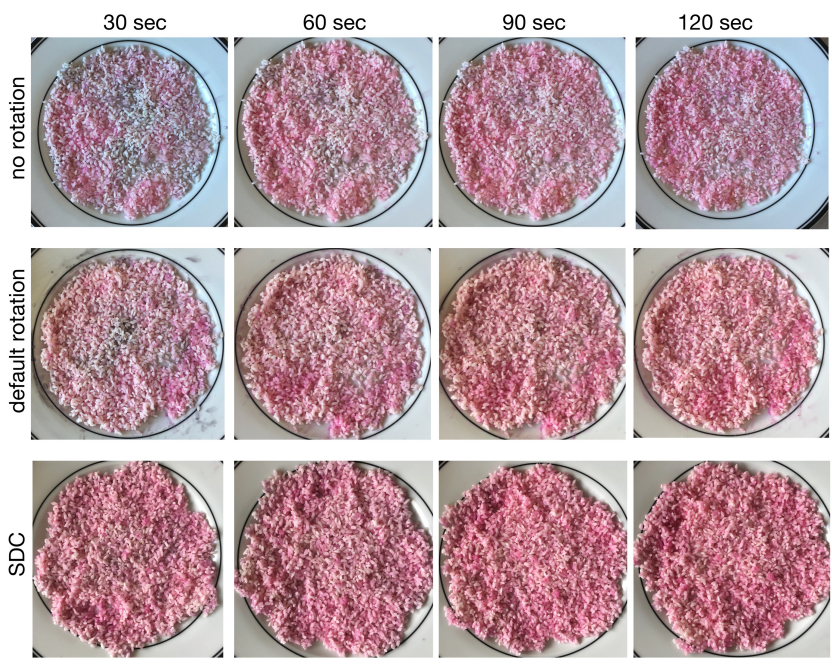

Figure 5: Visualization of heating of rice as a function of time for no rotation, default rotation and SDC. SDC results in the most uniform heating.

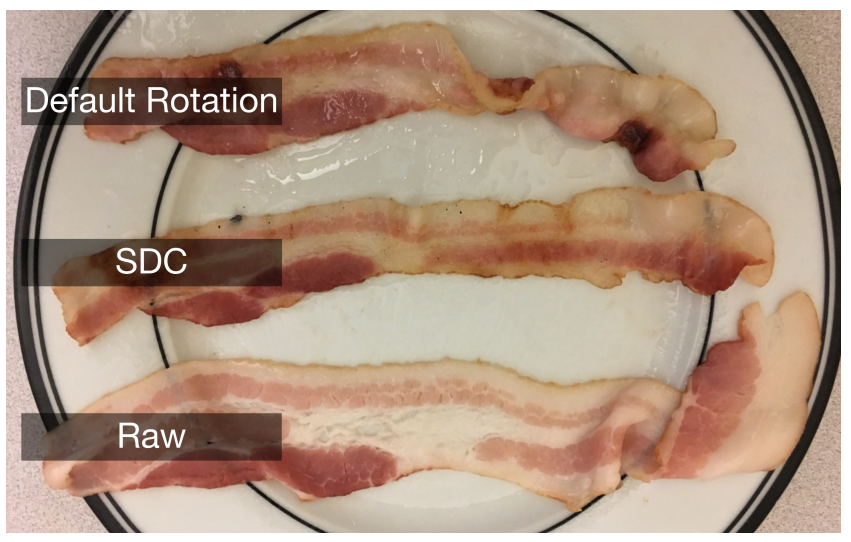

Figure 6: The raw bacon, and slices of bacon cooked with SDC and the original turntable. These three slices of bacon are from the same package, so the original fat patterns are nearly identical. Heated meat and fat will shrink. SDC applies heat to meat and fat discriminatively, so the fat shrinks more than the meat.

does not overcook the meat, while at the same time avoiding colder spots that may pose a health hazard.

\section{REFERENCES}

[1] Jin, H., Wang, J., Kumar, S., And Hong, J. Software defined cooking using a microwave oven. In Proceedings of the 25th Annual International Conference on Mobile Computing and Networking (New York, NY, USA, 2019), MobiCom '19, ACM.

[2] Potter, J. Cooking for geeks: real science, great hacks, and good food. OReilly, 2016.

[3] Public Broadcasting Service. The Mind of a Chef - Memory. Season 1 Episode 3. TV, 2012. 